Received: 20 February 2018

Accepted: 10 August 2018

Published online: 27 August 2018

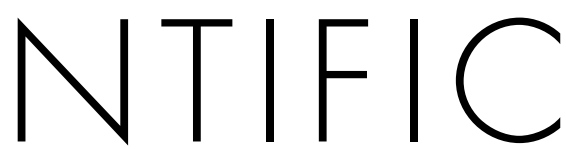

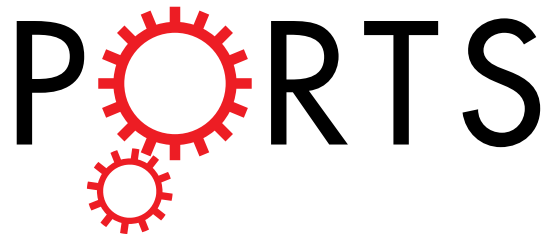

\title{
OPEN Vitamin D receptor Fokl polymorphism and the risks of colorectal cancer, inflammatory bowel disease, and colorectal adenoma
}

\begin{abstract}
Young Ae Cho $\mathbb{D}^{1}$, Jeonghee Lee $\mathbb{D}^{1}$, Jae Hwan $\mathrm{Oh}^{2}$, Hee Jin Chang ${ }^{2}$, Dae Kyung Sohn $\mathbb{D}^{2}$, Aesun $\mathrm{Shin}^{3}$ \& Jeongseon Kim ${ }^{1}$

Based on an inverse association between vitamin $D$ levels and the risks of colorectal diseases, a functional start codon polymorphism in the vitamin $D$ receptor (VDR) gene is speculated to affect the risks for these diseases. To validate this hypothesis, we first conducted a case-control study of 695 colorectal cancer patients and 1,397 controls. The association of VDR Fokl polymorphism with colorectal cancer risk was analyzed using a logistic regression model. In the present case-control study, compared to the $\mathrm{F}$ allele, the $\mathrm{f}$ allele seemed to be associated with lower risks of colon cancer and advanced colorectal cancer. Additionally, a meta-analysis of 27 studies was conducted to combine findings from previous studies investigating the association of Fokl polymorphism with colorectal disease using a random effects model. In the present meta-analysis, the $f$ allele was positively associated with the risk of inflammatory bowel disease, including Crohn's disease and ulcerative colitis. However, this allele was inversely associated with colon cancer and was not associated with the risk of rectal cancer or colorectal adenoma. In conclusion, the findings from this study imply that the role of VDR Fokl polymorphism may differ based on the type and severity of colorectal disease.
\end{abstract}

Epidemiologic studies have reported that low serum vitamin D levels are associated with an increased risk of colorectal diseases such as colorectal cancer $^{1}$, colorectal adenoma ${ }^{2}$, and inflammatory bowel disease ${ }^{3,4}$. The presence of colorectal adenoma and inflammatory bowel disease may increase the risk of colorectal cancer. Approximately $85 \%$ of colorectal cancer cases are thought to evolve from conventional adenomas through a process known as the adenoma-carcinoma sequence ${ }^{5}$. In addition, patients with inflammatory bowel disease have an increased risk of colorectal cancer compared to the general population ${ }^{6}$.

The active form of vitamin $\mathrm{D}, 1,25(\mathrm{OH})_{2} \mathrm{D}_{3}$, exerts its biological functions via the vitamin $\mathrm{D}$ receptor (VDR). Therefore, the protective effects of vitamin $\mathrm{D}$ against colonic carcinogenesis are most likely mediated through the VDR, which is expressed in approximately 30 different tissues. After binding to $1,25(\mathrm{OH})_{2} \mathrm{D}_{3}$, the VDR transactivates genes that modulate the immune response, inhibit proliferation, or promote differentiation and apopto$\mathrm{sis}^{7,8}$. Therefore, the associations of VDR polymorphisms with the risks of various diseases, including cancer and immune disease, have been extensively examined in epidemiologic studies. Among numerous polymorphisms identified in the VDR gene, the FokI (rs2228570) polymorphism is considered an independent marker because it has no linkage disequilibrium with any other VDR polymorphisms ${ }^{9}$. Additionally, this polymorphism changes the first start codon in the gene from ATG to ACG, resulting in a VDR protein that is shorter by three amino acids. The protein encoded by the FokI F allele (C allele) more efficiently binds vitamin $\mathrm{D}$ than the longer version coded by the $\mathrm{f}$ allele $(\mathrm{T} \text { allele })^{9,10}$. Therefore, the $\mathrm{f}$ allele may mimic the cellular consequences of lower vitamin $\mathrm{D}$ levels.

${ }^{1}$ Department of Cancer Biomedical Science, Graduate School of Cancer Science and Policy, National Cancer Center, Goyang, South Korea. ${ }^{2}$ Center for Colorectal Cancer, National Cancer Center Hospital, National Cancer Center, Goyang, South Korea. ${ }^{3}$ Department of Preventive Medicine, Seoul National University College of Medicine, Seoul, South Korea. Correspondence and requests for materials should be addressed to J.K. (email: jskim@ncc.re.kr) 
Previous studies have reported inconsistent findings regarding the associations of VDR FokI polymorphism with the risks of colorectal cancer, colorectal adenoma, and inflammatory bowel disease. Positive, negative, or no associations with these colorectal diseases have been reported ${ }^{11-14}$. Therefore, we hypothesized that the role of this polymorphism may differ by disease status. Since achieving sufficient statistical power to detect associations between polymorphisms and disease risks can be difficult in individual studies, a meta-analysis combining data from all published studies may detect genetic associations more accurately and reduce the probability of false-negative results ${ }^{15}$. Some meta-analyses have discussed the roles of FokI polymorphism ${ }^{16,17}$, but to the best of our knowledge, none have investigated its role in colorectal disease comprehensively.

The aim of this study was to investigate the association between VDR FokI polymorphism and the risk of colorectal disease. Therefore, a systematic meta-analysis was performed to combine data from previous studies on the association between VDR FokI polymorphism and the risks of colorectal diseases, including colorectal cancer, colorectal adenoma, and inflammatory bowel disease. To add more evidence, we also conducted a case-control study in Korea.

\section{Results}

Findings from the Present Case-Control Study. Compared to the controls, the cases were more likely to have a family history of colorectal cancer $(P<0.001)$, to be less educated $(P<0.001)$, to have a low level of regular exercise $(P<0.001)$, and to have a higher total caloric intake $(P<0.001)$. In contrast, no significant differences were identified between the cases and controls in terms of body mass index (BMI), smoking status, and alcohol consumption (Supplementary Table S1).

VDR FokI polymorphism was not associated with colorectal cancer. However, in a stratified analysis by anatomic location and cancer stage, FokI polymorphism was associated with colon cancer and advanced-stage colorectal cancer at a $P$-value level of 0.05 (Table 1). An association with a lower risk of colon cancer was observed among those carrying the FokI f allele (odds ratio $(\mathrm{OR})=0.75,95 \%$ confidence interval $(\mathrm{CI})=0.57-0.99$, $P=0.045$ for $\mathrm{Ff}$ vs. FF; $\mathrm{OR}=0.75,95 \% \mathrm{CI}=0.58-0.98, P=0.033$ for $\mathrm{Ff}+\mathrm{ff}$ vs. $\mathrm{FF})$; however, this association was not observed for rectal cancer. In addition, an association with a lower risk of colorectal cancer was observed for patients with advanced-stage colorectal cancer harboring the Fok $\mathrm{f}$ allele $(\mathrm{OR}=0.76,95 \% \mathrm{CI}=0.58-0.99$, $P=0.042$ for $\mathrm{Ff}$ vs. $\mathrm{FF} ; \mathrm{OR}=0.77,95 \% \mathrm{CI}=0.60-1.00, P=0.047$ for $\mathrm{Ff}+\mathrm{ff} \mathrm{vs}$. $\mathrm{FF})$.

Findings from the Meta-Analysis. Using our search criteria, a total of 101 articles were retrieved. After evaluating these articles, 76 were excluded for several reasons and 1 was added after screening the references of the retrieved publications and review papers. Therefore, we identified a total of 26 articles on the association between VDR FokI polymorphism and colorectal diseases and included the results from the current case-control study. Finally, 27 studies investigating various colorectal diseases were included in this meta-analysis: colorectal cancer (16 studies; 10,257 cases/12,492 controls) ${ }^{11,12,18-30}$, colorectal adenoma (4 studies; 1,322 cases/1,420 controls $)^{14,31-33}$, ulcerative colitis (6 studies; 1,555 cases/2,295 controls) ${ }^{13,34-38}$, and Crohn's disease (4 studies; 946 cases/1,390 controls) ${ }^{34-36,39}$. Three articles investigated both ulcerative colitis and Crohn's disease ${ }^{34-36}$. A study flowchart depicting the literature search and selection process is presented in Supplementary Fig. S1. Table 2 presents the characteristics of the studies included in the meta-analysis.

The meta-analysis was conducted to investigate the association between VDR FokI polymorphism and colorectal disease (Supplementary Table 2, Figs 1 and 2). VDR FokI polymorphism was not significantly associated with colorectal cancer risk. However, a borderline significant association with a lower risk of colon cancer was observed for heterozygous carriers compared to homozygous carriers of the $\mathrm{F}$ allele $(\mathrm{OR}=0.83,95 \%$ $\mathrm{CI}=0.69-1.00, P=0.049$ for $\mathrm{Ff}$ vs. FF). This polymorphism was not associated with rectal cancer. However, carriers of the $\mathrm{f}$ allele showed an increased risk of inflammatory bowel disease $(\mathrm{OR}=1.38,95 \% \mathrm{CI}=1.06-1.78$, $P=0.015$ for ff vs. FF; $\mathrm{OR}=1.32,95 \% \mathrm{CI}=1.17-1.50, P<0.001$ for $\mathrm{f}$ allele vs. F allele), both Crohn's disease $(\mathrm{OR}=1.46,95 \% \mathrm{CI}=1.08-1.98, P=0.015$ for $\mathrm{f}$ allele vs $\mathrm{F}$ allele $)$ and ulcerative colitis $(\mathrm{OR}=1.27,95 \% \mathrm{CI}=1.14-$ $1.41, P<0.001$ for f allele vs. F allele). No significant association was observed for colorectal adenoma $(\mathrm{OR}=1.11$, $95 \% \mathrm{CI}=0.99-1.23, P=0.077$ for $\mathrm{f}$ allele vs. $\mathrm{F}$ allele), although the direction of the association was similar to that for inflammatory bowel disease.

Significant heterogeneity was observed in the associations with colorectal cancer, colon cancer, inflammatory bowel disease, and Crohn's disease, as presented by Q-statistics (Supplementary Table 2) and I ${ }^{2}$ statistics (data not shown). Therefore, we conducted subgroup analyses and sensitivity analyses. When stratified by geographic location, the association between the $\mathrm{f}$ allele and colon cancer was stronger in studies conducted in non-Asian countries with low heterogeneity $(\mathrm{OR}=0.86,95 \% \mathrm{CI}=0.77-0.96, P=0.010$ for $\mathrm{Ff}$ vs. FF; $P$ for heterogeneity $=0.452)$. Generally, heterogeneity was lower in non-Asian studies (Supplementary Table 3). In sensitivity analyses, excluding the study of Naderi et al..$^{35}$ resulted in decreased heterogeneity in studies of Crohn's disease with a significant positive association $(\mathrm{OR}=1.26,95 \% \mathrm{CI}=1.10-1.45, P=0.001$ for $\mathrm{f}$ allele vs. F allele; $P$ for heterogeneity $=0.312)$. Additionally, excluding the study of Wong et al. ${ }^{18}$ resulted in a stronger inverse association and decreased the heterogeneity in colon cancer studies $(\mathrm{OR}=0.79,95 \% \mathrm{CI}=0.67-0.92, P=0.002$ for $\mathrm{Ff}$ vs. FF; $P$ for heterogeneity $=0.069)$. However, other sensitivity analyses did not show any meaningful changes in significance or heterogeneity (Supplementary Table S4).

Visual inspection of the funnel plot revealed no publication bias (data not shown). Additionally, the results of Egger's test indicated no significant publication bias (Supplementary Table 2).

\section{Discussion}

The present study including both a Korean case-control study and a meta-analysis of 27 studies suggested that VDR FokI polymorphism may have different associations with colorectal disease according to the type and severity of the disease. 


\begin{tabular}{|c|c|c|c|c|c|c|}
\hline \multirow[b]{2}{*}{ VDR FokI } & \multicolumn{2}{|c|}{ Number (\%) } & \multirow{2}{*}{$\begin{array}{l}\text { Crude OR } \\
(95 \% \text { CI })\end{array}$} & \multirow[b]{2}{*}{$P$-Value } & \multirow{2}{*}{$\begin{array}{l}\text { Adjusted OR } \\
(95 \% \mathrm{CI})^{(\mathrm{a})}\end{array}$} & \multirow[b]{2}{*}{$P$-value } \\
\hline & Controls & Cases & & & & \\
\hline \multicolumn{7}{|l|}{ Colorectal cancer } \\
\hline $\mathrm{FF}$ & $448(32.1)$ & $252(36.3)$ & 1.0 (ref) & & 1.0 (ref) & \\
\hline $\mathrm{Ff}$ & $697(49.9)$ & $315(45.3)$ & $0.80(0.66-0.99)$ & 0.035 & $0.83(0.66-1.04)$ & 0.104 \\
\hline $\mathrm{ff}$ & $252(18.0)$ & $128(18.4)$ & $0.90(0.69-1.17)$ & 0.447 & $0.92(0.68-1.24)$ & 0.591 \\
\hline $\mathrm{Ff}+\mathrm{ff}$ vs. FF & & & $0.83(0.69-1.01)$ & 0.056 & $0.85(0.69-1.06)$ & 0.145 \\
\hline $\mathrm{f}$ allele vs. F allele & & & $0.93(0.81-1.05)$ & 0.240 & $0.94(0.81-1.09)$ & 0.386 \\
\hline \multicolumn{7}{|l|}{ Tumor location } \\
\hline \multicolumn{7}{|l|}{ Colon cancer } \\
\hline $\mathrm{FF}$ & $448(32.1)$ & $137(38.9)$ & 1.0 (ref) & & 1.0 (ref) & \\
\hline Ff & 697 (49.9) & $157(44.6)$ & $0.74(0.57-0.95)$ & 0.020 & $0.75(0.57-0.99)$ & 0.045 \\
\hline $\mathrm{ff}$ & $252(18.0)$ & $58(16.5)$ & $0.75(0.53-1.06)$ & 0.105 & $0.75(0.52-1.09)$ & 0.134 \\
\hline $\mathrm{Ff}+\mathrm{ff}$ vs. FF & & & $0.74(0.58-0.94)$ & 0.015 & $0.75(0.58-0.98)$ & 0.033 \\
\hline fvs. F & & & $0.84(0.71-0.99)$ & 0.044 & $0.84(0.70-1.01)$ & 0.067 \\
\hline \multicolumn{7}{|l|}{ Rectal cancer } \\
\hline $\mathrm{FF}$ & $448(32.1)$ & $111(33.6)$ & 1.0 (ref) & & 1.0 (ref) & \\
\hline $\mathrm{Ff}$ & 697 (49.9) & $138(46.4)$ & $0.89(0.68-1.16)$ & 0.382 & $0.93(0.69-1.25)$ & 0.624 \\
\hline $\mathrm{ff}$ & $252(18.0)$ & $66(20.0)$ & $1.06(0.75-1.49)$ & 0.750 & $1.12(0.77-1.62)$ & 0.565 \\
\hline $\mathrm{Ff}+\mathrm{ff}$ vs. FF & & & $0.93(0.72-1.20)$ & 0.584 & $0.98(0.74-1.29)$ & 0.875 \\
\hline f vs. F & & & $1.01(0.85-1.20)$ & 0.927 & $1.04(0.86-1.25)$ & 0.688 \\
\hline \multicolumn{7}{|l|}{ AJCC stage } \\
\hline \multicolumn{7}{|l|}{ Stage $0+\mathrm{I}+\mathrm{II}$} \\
\hline FF & $448(32.1)$ & $74(32.6)$ & 1.0 (ref) & & 1.0 (ref) & \\
\hline $\mathrm{Ff}$ & 697 (49.9) & $107(47.1)$ & $0.93(0.68-1.28)$ & 0.653 & $0.87(0.61-1.23)$ & 0.426 \\
\hline $\mathrm{ff}$ & $252(18.0)$ & $46(20.3)$ & $1.11(0.74-1.65)$ & 0.624 & $1.05(0.68-1.62)$ & 0.834 \\
\hline $\mathrm{Ff}+\mathrm{ff}$ vs. FF & & & $0.98(0.72-1.32)$ & 0.874 & $0.92(0.66-1.27)$ & 0.597 \\
\hline fvs. F & & & $1.04(0.85-1.26)$ & 0.735 & $1.00(0.81-1.25)$ & 0.981 \\
\hline \multicolumn{7}{|l|}{ Stage III + IV } \\
\hline $\mathrm{FF}$ & $448(32.1)$ & $165(38.3)$ & 1.0 (ref) & & 1.0 (ref) & \\
\hline Ff & 697 (49.9) & $189(43.9)$ & $0.74(0.58-0.94)$ & 0.013 & $0.76(0.58-0.99)$ & 0.042 \\
\hline $\mathrm{ff}$ & $252(18.0)$ & 77 (17.9) & $0.83(0.61-1.13)$ & 0.240 & $0.82(0.58-1.17)$ & 0.275 \\
\hline $\mathrm{Ff}+\mathrm{ff}$ vs. FF & & & $0.76(0.61-0.95)$ & 0.017 & $0.77(0.60-1.00)$ & 0.047 \\
\hline fvs.F & & & $0.88(0.75-1.02)$ & 0.097 & $0.88(0.74-1.04)$ & 0.141 \\
\hline
\end{tabular}

Table 1. Association between VDR FokI polymorphism and the risk of colorectal cancer in the case-control

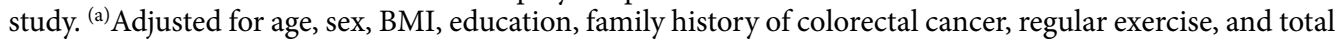
caloric intake. Abbreviations: AJCC, American Joint Committee on Cancer; CI, confidence interval; OR, odds ratio; VDR, vitamin D receptor.

To investigate the association between VDR FokI and colorectal cancer, we conducted both a case-control study and a meta-analysis of 16 previous studies. In the current case-control study, we found no association of the VDR FokI f allele with colorectal cancer. However, when cases were stratified by anatomic location and cancer stage, slightly inverse associations were observed with colon cancer and advanced-stage colorectal cancer. The inverse association of FokI polymorphism with colon or advanced-stage colorectal cancer was much stronger in a previous Korean study ${ }^{19}$ and was reported in other studies conducted in the USA ${ }^{11,28}$. Park et al. ${ }^{19}$ found that the f allele was less frequent among patients with advanced cancer stage and lymph node metastasis. However, Wong et al. ${ }^{18}$ reported the opposite association to other previous studies. In their study, the f allele was associated with an increased risk of colorectal cancer, but other studies reported positive or non-significant associations ${ }^{12,20-27,29,30}$. Therefore, to clarify these inconsistencies, we conducted a meta-analysis of 16 studies including the present case-control study. No significant association was observed, except for a borderline significant association with a lower risk of colon cancer among heterozygous carriers compared to homozygous carriers of the F allele. This association was stronger in studies conducted in non-Asian countries. The possible association with colon cancer but not rectal cancer was consistent with the findings of the present case-control study. The possible inverse association with colon cancer but not with rectal cancer, may derive from differences in the VDR distribution in these tissues ${ }^{40}$

The associations of FokI polymorphism with precancerous diseases such as inflammatory bowel disease and colorectal adenoma was examined in this meta-analysis. An increased risk of inflammatory bowel disease was observed among FokI f allele carriers compared to F allele carriers in the present meta-analysis. Several studies have reported significant positive associations of FokI polymorphism with Crohn's disease $\mathrm{s}^{35,36,39}$ and ulcerative colitis $^{13,35-38}$. In a Chinese case-control study ${ }^{38}$, the FokI f allele was more frequently observed in patients with severe ulcerative colitis than in those with mild or moderate ulcerative colitis. Additionally, several previous 


\begin{tabular}{|c|c|c|c|c|c|c|c|c|c|}
\hline \multirow[b]{2}{*}{ First author (year) } & \multirow[b]{2}{*}{ Country } & \multirow[b]{2}{*}{ Ethnicity } & \multirow[b]{2}{*}{ Disease } & \multirow[b]{2}{*}{$\begin{array}{l}\text { Control } \\
\text { source }\end{array}$} & \multicolumn{3}{|c|}{ Cases/Controls } & \multirow[b]{2}{*}{ MAF } & \multirow[b]{2}{*}{$\begin{array}{l}\text { Quality } \\
\text { Assessment }\end{array}$} \\
\hline & & & & & Number & Age (year) & \begin{tabular}{|l|}
$\begin{array}{l}\text { Female } \\
(\%)\end{array}$ \\
\end{tabular} & & \\
\hline Simmons $(2000)^{34}$ & UK & Caucasian & $\mathrm{UC}, \mathrm{CD}$ & $\mathrm{P}$ & $323 / 101$ & NR & $58 / \mathrm{NR}$ & 0.39 & 7 \\
\hline Ingles $(2001)^{14}$ & USA & $\begin{array}{l}\text { Caucasian, African, } \\
\text { Hispanic, Asian }\end{array}$ & $\mathrm{CA}$ & $\mathrm{P}$ & $373 / 394$ & $62.3 / 62.2$ & $32 / 34$ & 0.37 & 10 \\
\hline Peters $(2001)^{31}$ & USA & Caucasian, other & $\mathrm{CA}$ & $\mathrm{H}$ & $208 / 184$ & $60^{\mathrm{b}} / 57^{\mathrm{b}}$ & $23 / 37$ & 0.33 & 9 \\
\hline Wong $(2003)^{18}$ & Singapore & Asian $^{\mathrm{a}}$ & CRC & $\mathrm{P}$ & $217 / 890$ & $66 / 56.5$ & $42 / 57$ & 0.47 & 8 \\
\hline Murtaugh $(2006)^{11}$ & USA & $\begin{array}{l}\text { Caucasian, } \\
\text { Hispanic, African }\end{array}$ & $\mathrm{CRC}$ & $\mathrm{P}$ & $2450 / 2821$ & $30-79$ & $44 / 46$ & 0.38 & 10 \\
\hline Park $(2006)^{19}$ & Korea & Asian & CRC & $\mathrm{P}$ & $190 / 318$ & $55 / 55$ & $48 / \mathrm{NR}$ & 0.42 & 7 \\
\hline Flugge $(2007)^{20}$ & Germany & Caucasian & CRC & $\mathrm{H}$ & $256 / 256$ & \begin{tabular}{|l|}
$61.9 / 62.2$ \\
\end{tabular} & $52 / 51$ & 0.41 & 7 \\
\hline Grunhage $(2008)^{21}$ & Germany & Caucasian & CRC & $\mathrm{H}$ & $192 / 220$ & $65 / 63$ & $41 / 53$ & 0.32 & 7 \\
\hline Naderi $(2008)^{35}$ & Iran & NR & $\mathrm{UC}, \mathrm{CD}$ & $\mathrm{P}$ & $230 / 150$ & $35 / 35$ & $34 / 34$ & 0.26 & 7 \\
\hline Ochs-Balcom $(2008)^{22}$ & USA & Caucasian $^{(\mathrm{a})}$ & CRC & $\mathrm{P}$ & $250 / 246$ & $62.8 / 58.5$ & $52 / 67$ & 0.39 & 9 \\
\hline Theodoratou $(2008)^{23}$ & Scotland & Caucasian & CRC & $\mathrm{P}$ & $2940 / 3038$ & $62.0 / 62.4$ & $43 / 43$ & 0.39 & 9 \\
\hline Jenab $(2009)^{12}$ & Europe & Caucasian & CRC & $\mathrm{P}$ & $1248 / 1248$ & $58 / 58$ & $50 / 50$ & 0.39 & 10 \\
\hline Hughes $(2011)^{36}$ & Ireland & Caucasian & $\mathrm{UC}, \mathrm{CD}$ & $\mathrm{P}$ & $660 / 693$ & $41.2 / 40.2$ & $56 / 55$ & 0.35 & 7 \\
\hline Mahmoudi $(2011)^{24}$ & Iran & NR & CRC & $\mathrm{H}$ & $452 / 452$ & \begin{tabular}{|l|}
$53.8 / 44.3$ \\
\end{tabular} & $45 / 52$ & 0.25 & 7 \\
\hline Pei $(2011)^{13}$ & China & Asian $^{(\mathrm{a})}$ & UC & $\mathrm{P}$ & $218 / 250$ & $39.4 / 41.6$ & $35 / 44$ & 0.39 & 8 \\
\hline Bentley $(2012)^{25}$ & New Zealand & Caucasian & CRC & $\mathrm{P}$ & $200 / 200$ & 69.5 & $47 / 47$ & 0.38 & 6 \\
\hline Yamaji $(2012)^{32}$ & Japan & $\operatorname{Asian}^{(\mathrm{a})}$ & $\mathrm{CA}$ & $\mathrm{P}$ & $684 / 641$ & $40-79$ & $33 / 35$ & 0.34 & 9 \\
\hline Rasool $(2013)^{26}$ & India & NR & CRC & $\mathrm{P}$ & $312 / 305$ & \begin{tabular}{|l|}
$52.1 / 51.1$ \\
\end{tabular} & $45 / 49$ & 0.26 & 7 \\
\hline Laczmanska $(2014)^{27}$ & Poland & Caucasian $^{(\mathrm{a})}$ & CRC & $\mathrm{P}$ & $164 / 182$ & $65.7 / \mathrm{NR}$ & $41 / 41$ & 0.42 & 7 \\
\hline Sarkissyan $(2014)^{28}$ & USA & $\begin{array}{l}\text { Hispanic, African, } \\
\text { Caucasian, Asian }\end{array}$ & $\mathrm{CRC}$ & $\mathrm{H}$ & $78 / 230$ & $55.1 / 54.9$ & $45 / 63$ & 0.36 & 7 \\
\hline Takeshige (2015) ${ }^{29}$ & Japan & $\operatorname{Asian}^{(\mathrm{a})}$ & CRC & $\mathrm{P}$ & $685 / 778$ & \begin{tabular}{|l|}
$60.2 / 58.6$ \\
\end{tabular} & $38 / 37$ & 0.37 & 9 \\
\hline Xia $(2015)^{37}$ & China & Asian $^{(\mathrm{a})}$ & UC & $\mathrm{P}$ & $382 / 489$ & $42.1 / 41$ & $40 / 45$ & 0.43 & 7 \\
\hline Alkhayal $(2016)^{30}$ & Saudi Arabia & Saudi Arabian & CRC & $\mathrm{P}$ & $100 / 100$ & $57.5^{\mathrm{b}}$ & $36 / 35$ & 0.24 & 8 \\
\hline Beckett $(2016)^{33}$ & Australia & Caucasian $^{(\mathrm{a})}$ & $\mathrm{CA}$ & $\mathrm{P}$ & $57 / 201$ & $66.2 / 61.1$ & $46 / 59$ & 0.35 & 8 \\
\hline Xia $(2016)^{39}$ & \begin{tabular}{|l|} 
China \\
\end{tabular} & Asian $^{(\mathrm{a})}$ & $\mathrm{CD}$ & NR & $297 / 446$ & \begin{tabular}{|l|}
$27.1 / 28.2$ \\
\end{tabular} & $45 / 46$ & 0.43 & 7 \\
\hline Cho (2017, current) & Korea & Asian & CRC & $\mathrm{P}$ & $701 / 1402$ & \begin{tabular}{|l|}
$56.4 / 56.0$ \\
\end{tabular} & $32 / 32$ & 0.43 & 8 \\
\hline Zheng $(2017)^{38}$ & China & $\operatorname{Asian}^{(\mathrm{a})}$ & UC & $\mathrm{P}$ & $404 / 612$ & $42.1 / 40.8$ & $55 / 54$ & 0.43 & 7 \\
\hline
\end{tabular}

Table 2. Characteristics of the studies included in the meta-analysis. ${ }^{(a)}$ No information on race in the paper. The race was hypothesized based on the more frequent ethnicity in the study country; ${ }^{(b)}$ Median. Abbreviations: CA, colorectal adenoma; CD, Crohn's disease; CRC, colorectal cancer; $\mathrm{H}$, hospital-based; MAF, minor allele frequency (f allele); NR, not reported; P, population-based; UC, ulcerative colitis.

studies have examined the VDR FokI variant in relation to colorectal adenoma ${ }^{14,31-33}$, a precursor lesion for colorectal cancer. The present meta-analysis showed that VDR FokI polymorphism had no association with the risk of colorectal adenoma, although the direction of the association was similar to that for inflammatory bowel disease.

Inflammatory bowel disease is a chronic and non-specific inflammatory disease of the gastrointestinal tract resulting from inappropriate function of the mucosal immune system ${ }^{41}$. Vitamin D and VDR are suggested to protect the intestine from damage by maintaining epithelial barrier function and decreasing mucosal inflammation. The shorter allele $(\mathrm{F})$ of the FokI polymorphism was reported to be more efficient at transactivating vitamin D-target genes and is thus expected to transmit stronger anti-proliferative and pro-differentiation signals ${ }^{18}$. Therefore, the FokI $f$ allele may influence the immune-regulatory function of the $\mathrm{VDR}^{38,42}$. Based on this relationship, the less active ff genotype would be expected to mimic the cellular consequences of lower vitamin $\mathrm{D}$ levels and may therefore increase the risk of disease. Several studies ${ }^{6,43}$ reported an inverse correlation between the circulating $25(\mathrm{OH}) \mathrm{D}$ concentration and the risk of inflammatory bowel disease. Based on this information, the f allele may cause vitamin D deficiency, thus increasing the risk of inflammatory bowel disease. In the present study, the FokI f allele was positively associated with the risk of inflammatory bowel disease, which is consistent with the proposed mechanism.

However, the mechanism by which the FokI polymorphism influences susceptibility to colorectal cancer may be more complex and is poorly understood. The FokI f allele is suggested to be inversely associated with colon cancer and advanced colorectal cancer in the present case-control study and with colon cancer in the present meta-analysis, which is different from the findings for inflammatory bowel disease. Previous evidence suggests that the role of the FokI polymorphism may differ by disease severity. First, VDR expression is reported to differ according to disease status ${ }^{44,45}$. A case-control study examining Puerto Rican patients reported a relationship between serum vitamin D levels, colonic VDR expression, and histologic disease activity ${ }^{44}$. Although colonic VDR expression in normal mucosa is positively correlated with serum vitamin D levels, VDR expression was significantly depressed in sporadic dysplasia and colorectal cancer tissues compared to that in normal 


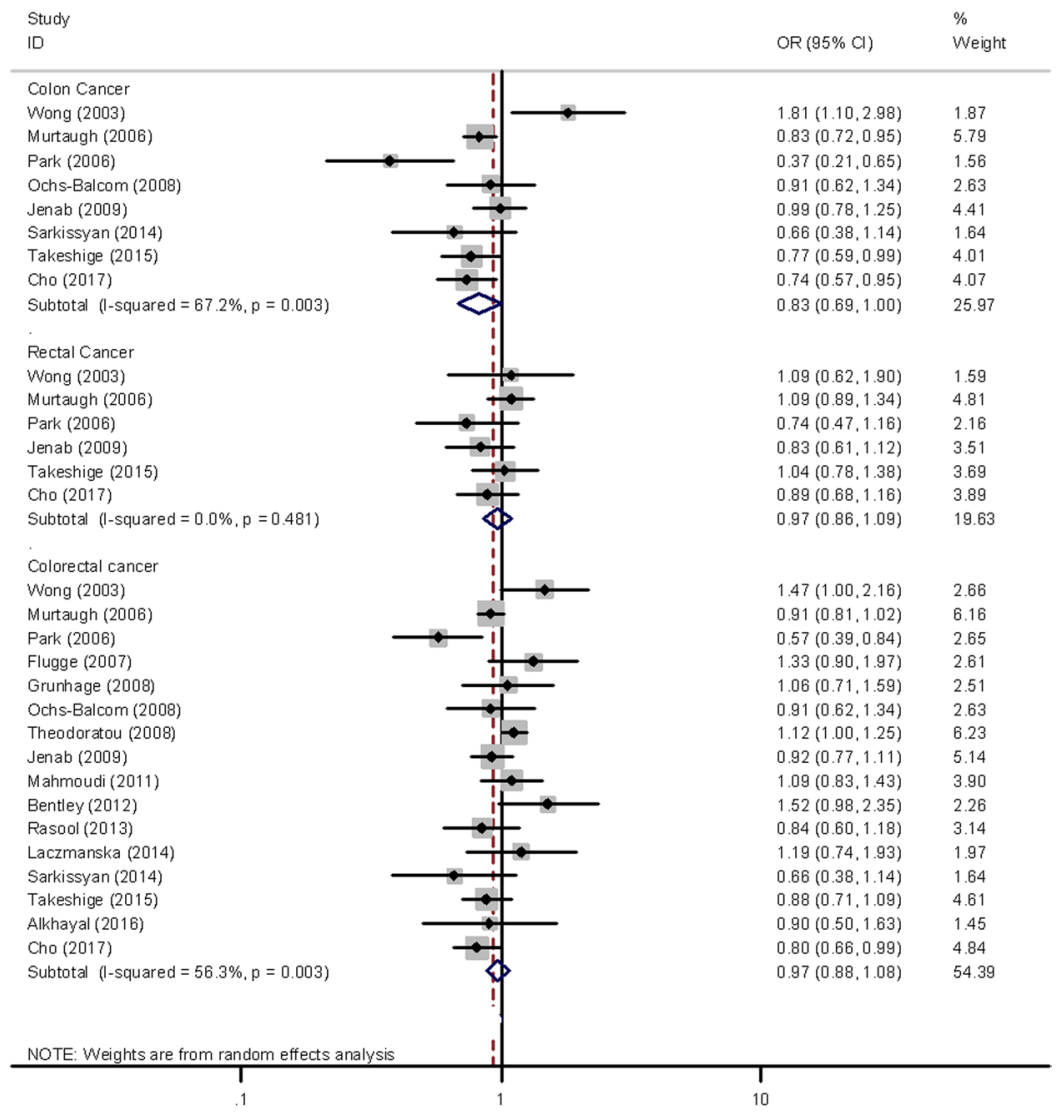

Figure 1. Forest plot showing the risk of colorectal cancer associated with VDR FokI polymorphism (Ft vs. FF).

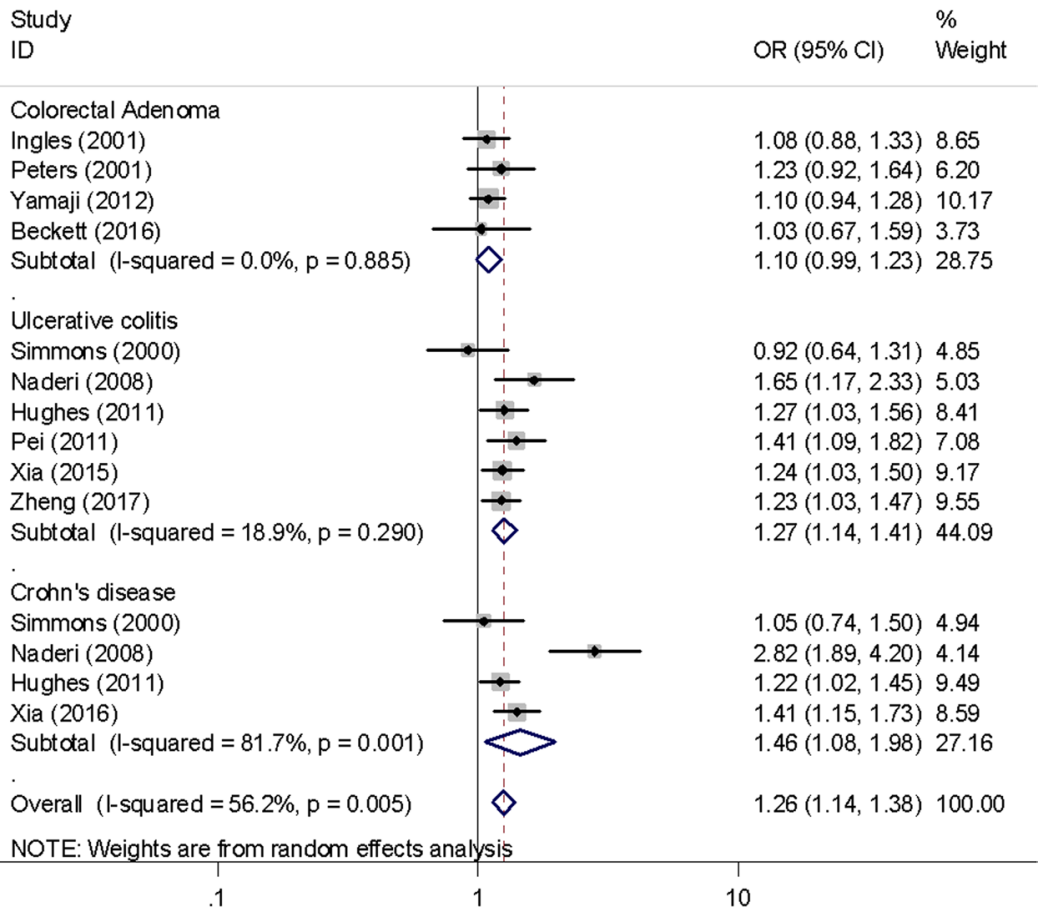

Figure 2. Forest plot showing the risk of colorectal adenoma and inflammatory bowel disease associated with VDR FokI polymorphism (f allele vs. F allele). 
and colitis-associated colorectal cancer tissues ${ }^{44,45}$. VDR expression in diseased colon tissues was associated with higher histologic inflammation scores, which reflect disease activity. Notably, the disease process interferes with the vitamin D-VDR system in diseased intestinal mucosa ${ }^{44}$. VDR expression has also been suggested to be up-regulated in early-stage cancer but not in advanced cancer ${ }^{19}$. Second, the role of vitamin D in carcinogenesis is influenced by tumor-host interactions. Although most vitamin D hydroxylation occurs in the liver and kidney, other tissues including intestinal epithelial cells and immune cells express vitamin D hydroxylase enzymes, suggesting that these cells can regulate local levels of the active hormone ${ }^{46}$. Because colorectal cancer with little lymphocytic infiltrate may not have sufficient bioactive vitamin $\mathrm{D}$, the inverse association between vitamin $\mathrm{D}$ and colorectal cancer may be stronger for cancers with high-level immune responses than that for cancer with low-level immune responses. A nested case-control study conducted in the USA ${ }^{47}$ reported that high plasma 25(OH)D levels are associated with a lower risk of colorectal cancer with an intense immune response. Therefore, host immunity and disease status may help predict the benefit of vitamin D and the role of the VDR in colorectal carcinogenesis. Future studies are required to elucidate the complex network that exists between VDR expression, $25(\mathrm{OH}) \mathrm{D}$ levels, FokI polymorphism, and patient health status.

The present case-control study has some limitations that should be considered when interpreting the findings. Because this study applied a case-control design, both recall and selection biases may be present. In the current case-control study, the controls were selected among those who voluntarily participated in a health check-up program; therefore, they may have been more health conscious than the general population. Additionally, subjects with inflammatory bowel disease and colorectal adenoma may have been included among the controls because the controls did not undergo endoscopy examination.

The meta-analysis also has several important limitations. First, the meta-analysis revealed significant heterogeneity for colorectal cancer, colon cancer, inflammatory bowel disease, and Crohn's disease but not for rectal cancer, ulcerative colitis, and colorectal adenoma. High heterogeneity across studies may undermine the strength of the findings. The different results between studies may have resulted from the clinical heterogeneity of colorectal disease, different demographic and genetic characteristics of the study populations, different sources of controls, different environment and lifestyle factors including vitamin D status, and the reliability of the genotyping data. To identify the cause of heterogeneity, we conducted subgroup analyses and sensitivity analyses; however, the exact sources of heterogeneity remain unclear. Second, the association with only one polymorphism was examined; therefore, other VDR polymorphisms should be considered in the future. Third, some inevitable publication bias may be present because only published studies were used, although no publication bias was observed. Fourth, this meta-analysis was based on unadjusted OR estimates because not all of the studies reported adjusted ORs. In addition, in studies in which adjusted ORs were presented, the ORs were not adjusted by the same potential confounders. Finally, the small number of studies included in the meta-analysis limits the ability to draw a significant conclusion, especially in subgroup analyses

In conclusion, we found that the role of the FokI polymorphism differs by the type and severity of colorectal disease. The FokI f allele was associated with an increased risk of inflammatory bowel disease and may be associated with a decreased risk of colon cancer. Based on this relationship, any impairment of the $1,25(\mathrm{OH})_{2} \mathrm{D}_{3} / \mathrm{VDR}$ system (e.g., vitamin D deficiency, VDR polymorphism, or impaired intestinal function) can be assumed to alter the development or progression of colorectal disease. Therefore, additional well-designed studies are needed to confirm the exact role of the VDR FokI polymorphism in colorectal diseases.

\section{Methods}

Case-Control Study. Study Subjects. This study has been described in detail elsewhere ${ }^{48}$. The eligible cases were newly diagnosed colorectal cancer patients at the Center for Colorectal Cancer of the National Cancer Center, Korea, between August 2010 and August 2013 who underwent surgery for colorectal cancer. Among the 1,070 patients who agreed to participate in the study, 369 were excluded because of incomplete semi-quantitative food frequency questionnaires (SQFFQs) or other questionnaires or implausible energy intakes $(<500$ or $>4,000 \mathrm{kcal} / \mathrm{day}$ ). Therefore, 923 patients were included in the analysis. The control subjects were recruited between October 2007 and December 2014 among individuals visiting the Center for Cancer Prevention and Detection at the same hospital for a health check-up program provided by the National Health Insurance Cooperation, which covers the entire Korean population. Of the 14,201 subjects who agreed to participate in the study, 5,164 were excluded because of incomplete SQFFQs or other questionnaires and implausible energy intakes ( $<500$ or $>4,000 \mathrm{kcal} /$ day). The data of the remaining subjects were linked with the Korea Central Cancer Registry and National Cancer Center medical chart to confirm that these subjects had not been diagnosed with colorectal cancer. Of the remaining 9,037 individuals, two controls per case were randomly selected and frequency-matched by sex and 5-year age group. Therefore, 923 cases and 1,846 controls were originally selected to investigate the association between environmental factors and colorectal cancer risk. However, among 923 cases, 222 were missing blood samples. Therefore, 1:2 matching was conducted again, and 701 cases and 1,402 controls were selected for genotyping. After genotyping, 6 cases and 5 controls were excluded because of genotyping failure. Ultimately, 695 colorectal cancer patients and 1,397 healthy controls were selected for the final analysis (Supplementary Fig. S2).

All participants provided written informed consent, and the study protocol was approved by the Institutional Review Board of the National Cancer Center (IRB No. NCCNCS-10-350 and NCC2015-0202). All procedures used in the present study were performed in accordance with the guidelines and regulations of the IRB of the National Cancer Center.

Data Collection and Genotyping. A trained interviewer conducted face-to-face interviews to collect information on lifestyle factors and dietary habits prior to cancer diagnosis. Information regarding the participants' 
demographic and lifestyle risk factors (e.g., smoking, alcohol drinking, and regular exercise) was collected using a structured questionnaire.

The VDR FokI polymorphism (rs2228570) was genotyped as described below. Genomic DNA was extracted using the MagAttract DNA Blood M48 kit (Qiagen, Hilden, Germany) and BioRobot M48 automatic extraction equipment (Qiagen) according to the manufacturer's instructions. Genotyping was performed using a MassARRAY iPLEX Gold Assay (Agena Bioscience, San Diego, CA). To control genotyping quality, duplicate samples for $3 \%$ of the subjects were included in our initial genotyping analysis; the rate of discordance was $<1 \%$. Genotyping was successfully performed for 695 cases and 1,397 controls.

Statistical analysis. Differences in the demographic and lifestyle factors between the cases and controls were analyzed using the $\chi^{2}$ test for categorical variables and Student's t-test for continuous variables. The $\chi^{2}$ test was used to test for Hardy-Weinberg Equilibrium (HWE) of the VDR FokI polymorphism in the control group. The association between VDR FokI polymorphism and colorectal cancer risk was analyzed using unconditional logistic regression models. A multivariable model was adjusted for age (continuous), sex (men/women), body mass index (BMI) $\left(<25, \geq 25 \mathrm{~kg} / \mathrm{m}^{2}\right)$, education (middle school or less, high school, college or more), family history of colorectal cancer (yes/no), regular exercise (yes/no), and total caloric intake (continuous). To determine which variables to enter into the multivariable model, we performed backward selection using colorectal risk factors, which were selected based on both our data and prior information. A multinomial logistic regression model was used for analyses stratified by anatomic location (colon and rectal cancer) and cancer stage. American Joint Committee on Cancer (AJCC) stages III and IV were considered advanced cancer.

All statistical analyses were performed using SAS 9.2 (SAS Institute Inc., Cary, NC). A two-sided $P$-value of less than 0.05 indicated statistical significance.

Meta-analysis of Published Studies. Literature Search. This meta-analysis was performed according to the PRISMA guidelines. To identify all articles exploring the association between VDR FokI polymorphism and the risk of colorectal cancer, colorectal adenoma, or inflammatory bowel disease, we conducted a literature search using PubMed and EMBASE through March 2017. We used the following search terms: (1) "VDR polymorphism", rs2228570, rs10735810, or FokI; (2) "colorectal cancer", "colon cancer", "rectal cancer", "colorectal adenoma", "inflammatory bowel disease", "ulcerative colitis" or "Crohn's disease". Search terms included both $\mathrm{MeSH}$ terms and text words. To identify additional studies, we also screened the references of the retrieved publications and review papers. This search was limited to human studies and publications written in English. We did not consider abstracts or unpublished reports.

Inclusion Criteria. The studies included in this meta-analysis were required to meet the following criteria: (1) investigated the association between VDR FokI polymorphism and the abovementioned diseases; (2) provided genotype frequencies for cases and controls such that ORs with 95\% CIs and HWE could be calculated; and (3) featured a control population genotype distribution that did not deviate from $\operatorname{HWE}(P>0.01)$. If duplicated data were reported in multiple publications, the most complete publication was selected.

Data Extraction and Quality Assessment. The following information was extracted from each article: author name, year of publication, country of study, ethnicity, source of the controls, mean age, proportion of women, vitamin D status, and genotype frequencies for cases and controls.

Predefined criteria (Supplementary Table S5) based on the scale of Thakkinstian et al. ${ }^{49}$ were used to assess the methodological quality of eligible studies (Supplementary Table S6). The revised criteria include the representativeness of cases and controls, assessment of colorectal disease, genotyping examination, HWE deviation in the control population, and association assessment. Scores ranged from 0 (lowest) to 11 (highest). Articles with scores of less than 6 were considered to be low-quality studies, whereas those with scores equal to or higher than 6 were considered high-quality reports. Data extraction and quality assessment were performed by two investigators independently. Disagreements were resolved by consensus.

Statistical analysis. The strengths of associations between VDR FokI polymorphism and selected disease risk (colorectal cancer, colorectal adenoma, and inflammatory bowel disease) were measured using unadjusted ORs with corresponding 95\% CIs, which were calculated based on genotype frequencies. Forest plots were used to illustrate the results of the included studies. Four different ORs were calculated using the following models: (1) homozygote comparison, (2) heterozygote comparison, (3) dominant genetic model, and (4) allele comparison. Before analysis, the genotype frequencies of the polymorphisms were assessed for HWE using the chi-squared test. A $P$-value less than 0.01 indicated a significant deviation from HWE. The summary OR estimated for each study was calculated using a random effects model. Crohn's disease and ulcerative colitis were assessed together or separately. Heterogeneity among the included articles was estimated using the Q-statistic and $I^{2}$ statistic. Potential sources of heterogeneity were sought via subgroup analyses by geographic location and anatomic location. We also performed sensitivity analyses by excluding one study each. Publication bias was assessed by visual inspection of funnel plots and formal statistical assessment of funnel plot asymmetry was performed with Egger's regression test. Publication bias was considered present if the $P$-value of the intercept was less than 0.05 .

All statistical analyses were performed using STATA software (version 14; Stata Corporation, College Station, Texas). Two-sided $P$-values less than 0.05 indicated statistical significance.

Data availability. The dataset generated and/or analyzed during the present study are available from the corresponding author on reasonable request. 


\section{References}

1. Giovannucci, E. Epidemiology of vitamin D and colorectal cancer. Anti-cancer Agents Med Chem. 13, 11-19 (2013).

2. Choi, Y. J., Kim, Y. H., Cho, C. H., Kim, S. H. \& Lee, J. E. Circulating levels of vitamin D and colorectal adenoma: A case-control study and a meta-analysis. World J Gastroenterol. 21, 8868-8877 (2015).

3. Mouli, V. P. \& Ananthakrishnan, A. N. Review article: vitamin D and inflammatory bowel diseases. Aliment Pharmacol Ther. 39, 125-136 (2014).

4. Ananthakrishnan, A. N. et al. Higher predicted vitamin D status is associated with reduced risk of Crohn's disease. Gastroenterology. 142, 482-489 (2012).

5. Strum, W. B. Colorectal adenomas. N Engl J Med. 375, 389-390 (2016).

6. Ananthakrishnan, A. N. et al. Association between reduced plasma 25-hydroxy vitamin D and increased risk of cancer in patients with inflammatory bowel diseases. Clin Gastroenterol Hepatol. 12, 821-827 (2014).

7. Kallay, E. et al. Characterization of a vitamin D receptor knockout mouse as a model of colorectal hyperproliferation and DNA damage. Carcinogenesis. 22, 1429-1435 (2001)

8. Mathiasen, I. S., Lademann, U. \& Jaattela, M. Apoptosis induced by vitamin D compounds in breast cancer cells is inhibited by Bcl2 but does not involve known caspases or p53. Cancer Res. 59, 4848-4856 (1999).

9. Uitterlinden, A. G., Fang, Y., Van Meurs, J. B., Pols, H. A. \& Van Leeuwen, J. P. Genetics and biology of vitamin D receptor polymorphisms. Gene. 338, 143-156 (2004).

10. Miyamoto, K. et al. Structural organization of the human vitamin D receptor chromosomal gene and its promoter. Mol Endocrinol. 11, 1165-1179 (1997).

11. Murtaugh, M. A. et al. Vitamin D receptor gene polymorphisms, dietary promotion of insulin resistance, and colon and rectal cancer. Nutr Cancer. 55, 35-43 (2006).

12. Jenab, M. et al. Vitamin D receptor and calcium sensing receptor polymorphisms and the risk of colorectal cancer in European populations. Cancer Epidemiol Biomarkers Prev. 18, 2485-2491 (2009).

13. Pei, F. H. et al. Vitamin D receptor gene polymorphism and ulcerative colitis susceptibility in Han Chinese. J Dig Dis. 12, 90-98 (2011).

14. Ingles, S. A. et al. Vitamin D receptor polymorphisms and risk of colorectal adenomas (United States). Cancer Causes Control. 12, 607-614 (2001).

15. Kavvoura, F. K. \& Ioannidis, J. P. Methods for meta-analysis in genetic association studies: a review of their potential and pitfalls. Hum Genet. 123, 1-14 (2008).

16. Gnagnarella, P. et al. Vitamin D receptor polymorphism FokI and cancer risk: a comprehensive meta-analysis. Carcinogenesis. 35 , 1913-1919 (2014).

17. Wang, L. et al. Polymorphisms of the vitamin D receptor gene and the risk of inflammatory bowel disease: a meta-analysis. Genet Mol Res. 13, 2598-2610 (2014).

18. Wong, H. L. et al. Vitamin D receptor start codon polymorphism and colorectal cancer risk: effect modification by dietary calcium and fat in Singapore Chinese. Carcinogenesis. 24, 1091-1095 (2003).

19. Park, K., Woo, M., Nam, J. \& Kim, J. C. Start codon polymorphisms in the vitamin D receptor and colorectal cancer risk. Cancer Lett. 237, 199-206 (2006).

20. Flugge, J. et al. Vitamin D receptor haplotypes protect against development of colorectal cancer. Eur J Clin Pharmacol. 63, 997-1005 (2007).

21. Grunhage, F. et al. Association of familial colorectal cancer with variants in the E-cadherin (CDH1) and cyclin D1 (CCND1) genes. Int J Colorectal Dis. 23, 147-154 (2008).

22. Ochs-Balcom, H. M. et al. Association of vitamin D receptor gene variants, adiposity and colon cancer. Carcinogenesis. 29, 1788-1793 (2008).

23. Theodoratou, E. et al. Modification of the inverse association between dietary vitamin D intake and colorectal cancer risk by a FokI variant supports a chemoprotective action of Vitamin D intake mediated through VDR binding. Int J Cancer. 123, 2170-2179 (2008).

24. Mahmoudi, T., Karimi, K., Mohebbi, S. R., Fatemi, S. R. \& Zali, M. R. Start codon FokI and intron 8 BsmI variants in the vitamin D receptor gene and susceptibility to colorectal cancer. Mol Biol Rep. 38, 4765-4770 (2011).

25. Bentley, R. W. et al. Vitamin D receptor polymorphisms in colorectal cancer in New Zealand: an association study. $N Z$ Med J. 125, 47-51 (2012)

26. Rasool, S. et al. Association of a VDR gene polymorphism with risk of colorectal cancer in Kashmir. Asian Pac J Cancer Prev. 14, 5833-5837 (2013).

27. Laczmanska, I. et al. Vitamin D receptor gene polymorphisms in relation to the risk of colorectal cancer in the Polish population. Tumour Biol. 35, 12397-12401 (2014).

28. Sarkissyan, M. et al. Vitamin D receptor FokI gene polymorphisms may be associated with colorectal cancer among African American and Hispanic participants. Cancer. 120, 1387-1393 (2014).

29. Takeshige, N. et al. Associations between vitamin D receptor (VDR) gene polymorphisms and colorectal cancer risk and effect modifications of dietary calcium and vitamin D in a Japanese population. Asian Pac J Cancer Prev. 16, 2019-2026 (2015).

30. Alkhayal, K. A. et al. Association of vitamin D receptor gene polymorphisms with colorectal cancer in a Saudi Arabian population. Plos One 11, e0155236, https://doi.org/10.1371/journal.pone.0155236 (2016).

31. Peters, U. et al. Vitamin D, calcium, and vitamin D receptor polymorphism in colorectal adenomas. Cancer Epidemiol Biomarkers Prev. 10, 1267-1274 (2001).

32. Yamaji, T. et al. Association between plasma 25-hydroxyvitamin D and colorectal adenoma according to dietary calcium intake and vitamin D receptor polymorphism. Am J Epidemiol. 175, 236-244 (2012).

33. Beckett, E. L. et al. Vitamin D receptor polymorphisms relate to risk of adenomatous polyps in a sex-specific manner. Nutr Cancer. 68, 193-200 (2016).

34. Simmons, J. D., Mullighan, C., Welsh, K. I. \& Jewell, D. P. Vitamin D receptor gene polymorphism: association with Crohn's disease susceptibility. Gut. 47, 211-214 (2000).

35. Naderi, N. et al. Association of vitamin D receptor gene polymorphisms in Iranian patients with inflammatory bowel disease. $J$ Gastroenterol Hepatol. 23, 1816-1822 (2008).

36. Hughes, D. J., McManus, R., Neary, P., O’Morain, C. \& O’Sullivan, M. Common variation in the vitamin D receptor gene and risk of inflammatory bowel disease in an Irish case-control study. Eur J Gastroenterol Hepatol. 23, 807-812 (2011).

37. Xia, S. L. et al. Association of vitamin D receptor gene polymorphisms with the susceptibility to ulcerative colitis in patients from Southeast China. J Recept Signal Transduct Res. 35, 530-535 (2015).

38. Zheng, S. Z. et al. The association between vitamin D receptor polymorphisms and serum 25-hydroxyvitamin D levels with ulcerative colitis in Chinese Han population. Clin Res Hepatol Gastroenterol. 41, 110-117 (2017).

39. Xia, S. L. et al. Association of vitamin D receptor gene polymorphisms and serum 25-hydroxyvitamin D levels with Crohn's disease in Chinese patients. J Gastroenterol Hepatol. 31, 795-801 (2016).

40. Wang, Y., Zhu, J. \& DeLuca, H. F. Where is the vitamin D receptor? Arch Biochem Biophys. 523, 123-133 (2012).

41. Podolsky, D. K. Inflammatory bowel disease. N England J Med. 347, 417-429 (2002). 
42. van Etten, E. et al. The vitamin D receptor gene FokI polymorphism: functional impact on the immune system. Eur J Immunol. 37, 395-405 (2007)

43. Sadeghian, M., Saneei, P., Siassi, F. \& Esmaillzadeh, A. Vitamin D status in relation to Crohn's disease: meta-analysis of observational studies. Nutrition. 32, 505-514 (2016).

44. Abreu-Delgado, Y. et al. Serum vitamin D and colonic vitamin D receptor in inflammatory bowel disease. World J Gastroenterol. 22, 3581-3591 (2016).

45. Isidro, R. A. et al. Immunohistochemical expression of SP-NK-1R-EGFR pathway and VDR in colonic inflammation and neoplasia. World J Gastroenterol. 21, 1749-1758 (2015).

46. Bikle, D. D. Vitamin D metabolism, mechanism of action, and clinical applications. Chem Biol. 21, 319-329 (2014).

47. Song, M. et al. Plasma 25-hydroxyvitamin D and colorectal cancer risk according to tumour immunity status. Gut. 65, 296-304 (2016).

48. Cho, Y. A. et al. Dietary flavonoids, CYP1A1 genetic variants, and the risk of colorectal cancer in a Korean population. Sci Rep. 7, 128, https://doi.org/10.1038/s41598-017-00117-8 (2017).

49. Thakkinstian, A. et al. Systemic review and meta-analysis of the association between $\beta_{2}$-adrenoceptor polymorphisms and asthma: a HuGE review. Am J Epidemiol. 162, 201-211 (2005).

\section{Acknowledgements}

This work was supported by grants from the National Cancer Center in Korea $(1510040,1710882)$, the National Research Foundation of Korea (2015R1C1A2A01053728, and 2015R1A5A6001906).

\section{Author Contributions}

Y.A.C., A.S. and J.K. designed and conducted the research and revised the manuscript. Y.A.C. performed the statistical analysis and drafted the manuscript. Y.A.C. and J.K. conducted the literature search and collected data for the meta-analysis. J.L., J.H.O., H.J.C. and D.K.S. recruited the study subjects and collected the data. All authors read and approved the final manuscript.

\section{Additional Information}

Supplementary information accompanies this paper at https://doi.org/10.1038/s41598-018-31244-5.

Competing Interests: The authors declare no competing interests.

Publisher's note: Springer Nature remains neutral with regard to jurisdictional claims in published maps and institutional affiliations.

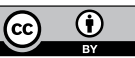

Open Access This article is licensed under a Creative Commons Attribution 4.0 International License, which permits use, sharing, adaptation, distribution and reproduction in any medium or format, as long as you give appropriate credit to the original author(s) and the source, provide a link to the Creative Commons license, and indicate if changes were made. The images or other third party material in this article are included in the article's Creative Commons license, unless indicated otherwise in a credit line to the material. If material is not included in the article's Creative Commons license and your intended use is not permitted by statutory regulation or exceeds the permitted use, you will need to obtain permission directly from the copyright holder. To view a copy of this license, visit http://creativecommons.org/licenses/by/4.0/.

(C) The Author(s) 2018 